\title{
Poling-inhibited ridge waveguides in lithium niobate crystals
}

\author{
C. L. Sones ${ }^{1, *}$, P. Ganguly ${ }^{2}$, C. Y. J. Ying ${ }^{1}$, E. Soergel ${ }^{3}$, R. W. Eason ${ }^{1}$, S. Mailis ${ }^{1}$ \\ ${ }^{1}$ Optoelectronics Research Centre, University of Southampton, Southampton, SO17 1BJ, United Kingdom \\ ${ }^{2}$ Advanced Technology Development Centre, Indian Institute of Technology, Kharagpur, 721302, India \\ ${ }^{3}$ Institute of Physics, University of Bonn, Wegelerstr. 8, 53115 Bonn, Germany
}

\begin{abstract}
Ultraviolet laser irradiation of a lithium niobate $+z$ polar surface enables the production of ridge waveguides. Ultraviolet laser induced inhibition of poling is used to define an inverted domain pattern which transforms into a ridge structure by differential etching in Hydrofluoric acid. The laser irradiation step also induces a refractive index change that provides the vertical confinement within the ridge structure. Furthermore, it was observed that poling-inhibition results in a significant enhancement of the refractive index contrast between the bulk crystal and the ultraviolet irradiated tracks.
\end{abstract}

Lithium niobate $(\mathrm{LN})$ because of its distinctive combination of inherent physical properties; has always been recognised as an appropriate platform for implementation of integrated optical circuits ${ }^{1}$. One of the suggested approaches which is quite regularly implemented to further improve the efficiency and compactness of such integrated optical circuits based on components such as modulators and resonators, is the use of ridge waveguides instead of conventional diffused ${ }^{2}$ or proton-exchanged $^{3}$ waveguides. Such ridge waveguide superstructures provide better lateral confinement of the optical mode due to the much higher index contrast as compared to their conventional counterparts.

Several different methods have been investigated for the production of ridge waveguides in LN. Most of them utilize an etching step that defines the ridge geometry and a separate waveguide fabrication step ${ }^{4-6}$ such as the commonly used ionindiffusion or proton-exchange processes. Delineation of the ridge is achieved either via wet etching in an acid mixture containing hydrofluoric acid (HF) or through dry etching processes such as ion beam milling ${ }^{7}$ or plasma etching ${ }^{8}$. Domain-selective acid-based wet etching has also been reported as an alternative approach for defining ridges in domain engineered $\mathrm{LN}^{9}$. Interestingly, ridge waveguides have also been fabricated by mechanical dicing of planar $\mathrm{MgO}: \mathrm{LN}$ bonded on to $\mathrm{LN}^{10}$. The mandatory step required to induce the vertical refractive index (RI) contrast either precedes or succeeds the wet or dry etching process. In all of these approaches, a clean-room based photolithographic step is incorporated to define the ridge on an already produced planar waveguide or to define the waveguide on a prestructured ridge.

Any alternative process that reduces the complexity of fabrication would be extremely well-suited for the implementation of the dense compact photonic circuits envisaged for future optoelectronic integration technologies. In this contribution we report on such an alternative method for the fabrication of ridge waveguides in $z$-cut $L N$. The method utilizes a continuous wave (c.w.) UV laser direct writing procedure that provides both the definition of the ridge pattern and the production of the necessary RI contrast for vertical confinement in a single step. Direct writing (DW) of waveguides in congruent LN has been demonstrated using c.w. UV laser light with writing wavelengths within the range of $244 \mathrm{~nm}-305 \mathrm{~nm}{ }^{11,12}$, which are below the UV absorption edge of the material. Furthermore, this initial UV exposure of the $+z$ face of $\mathrm{LN}$ crystals is reported to inhibit domain inversion locally during subsequent electric field poling (EFP) ${ }^{13}$. As a result a ferroelectric domain structure corresponding to the UV laser irradiated tracks is formed after EFP. Subsequent differential wet etching of these polinginhibited domain structures results in the creation of surfacerelief structures such as ridges, which can support optical guided-wave propagation because of the already inscribed RI change. Of course, the method allows for a straightforward fabrication of arbitrary patterns as allowed by the direct laser writing, which makes it particularly attractive for custom fabrication of photonic microstructures. In this communication we demonstrate the fabrication of simple waveguide ridges as a proof of principle.

A schematic of the fabrication procedure detailing the processing steps that leads to the formation of the ridge waveguides is shown in Fig. 1. The first step in the sequence involves the inscription of a RI change in LN using the UV laser DW procedure. A beam from an Ar-ion laser $(\lambda=275 \mathrm{~nm})$, focused to a spot radius of $\sim 3 \mu \mathrm{m}$, was used for the local irradiation of the $+z$ face of a congruently melting, undoped LN crystal. Precise control over the positioning and exposure of the illuminating laser beam was achieved by a computer-controlled, three-axis stage system coupled with a mechanical shutter. Sets of parallel lines were drawn on the $+z$ faces of the crystals along the crystallographic $y$ direction by moving the stages at speeds ranging from 0.1 to $1 \mathrm{~mm} / \mathrm{s}$. The incident intensity was varied between $0.1-$ $0.25 \mathrm{MW} / \mathrm{cm}^{2}$.

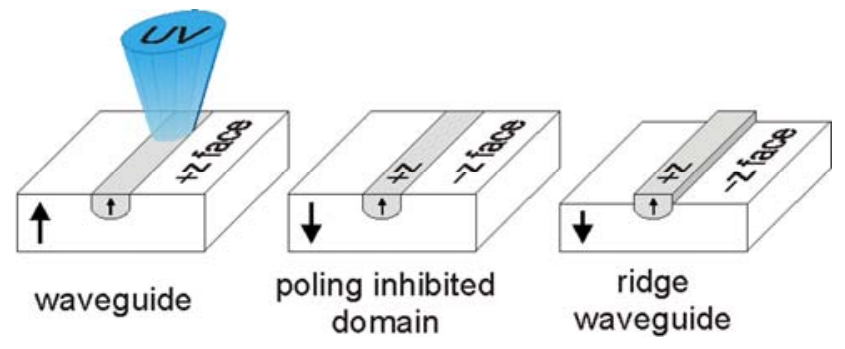

FIG. 1 Schematic of the fabrication process showing the three sequential fabrication steps of UV patterning, EFP, and wet-etching. 


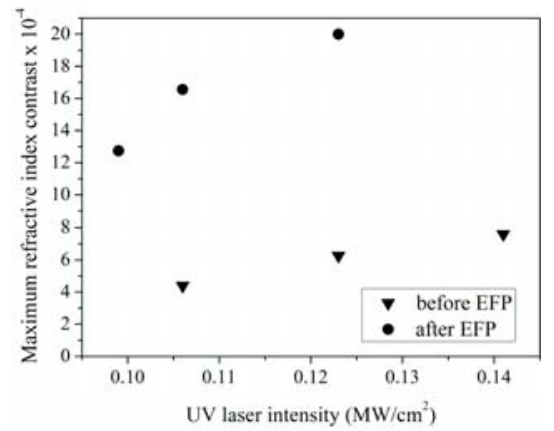

FIG 2. Plot showing variation of Max RI change before and after EFP.

The second step incorporates EFP to transform the UV written pattern into the corresponding domain pattern via poling inhibition. Samples with the sets of UV exposed parallel lines were poled using the EFP set-up described in Ref. 13. The sample was initially conditioned by several poling cycles before the final forward poling step. In this final poling step, the voltage was ramped at $0.1 \mathrm{kV} / \mathrm{s}$ to a value of $10 \mathrm{kV}$ applied across the $0.5 \mathrm{~mm}$ thick sample. Conditioning of the crystal allows poling to occur at this lower voltage (as compared to the virgin crystal) but most importantly promotes a much slower domain wall motion which is desirable since the kinetics of the domain wall motion are seen to greatly influence the shape, quality and more essentially the continuity of the resultant ridge structures. As described in Ref. 13, the UV exposed areas (the linear tracks in our case) of the $+z$ face, resist domain inversion during subsequent EFP retaining their original $(+z)$ polarity whereas the rest of the un-illuminated crystal undergoes domain inversion. As shown in Fig. 1, the result is a set of parallel stripes of $+z$ polar surface embedded in a uniform $-z$ polar surface background.

It has been reported in the literature that a RI increase is observed in the newly poled region as a consequence of EFP. This RI increase has been measured using digital holography ${ }^{14}$ and scanning near-field optical microscopy ${ }^{15}$ imaging and is attributed to the internal field which originates from the presence of frustrated defect clusters in the newly inverted domain region. An enhancement of the RI of the newly poled area encapsulating the waveguide channel (as in the case of the poling inhibition) is expected to affect negatively the performance of the waveguide by reducing the RI contrast between the channel and this newly poled substrate region. The impact of the EFP step on the UV written waveguide channels was investigated using the method described in Ref. 12.



FIG. 3 Optical microscope image of two sets of ridges produced using different UV laser intensities.

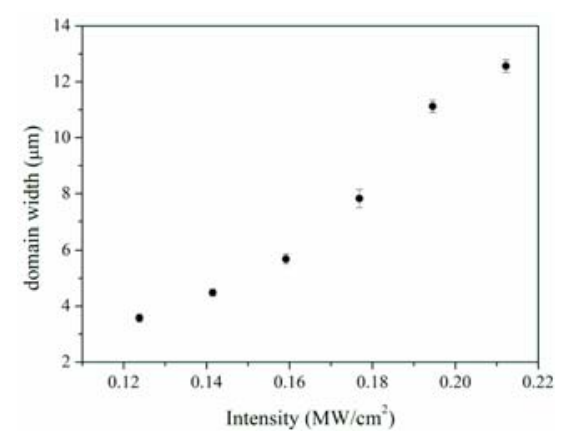

FIG. 4 Plot of the poling inhibited domain width as a function of the UV laser $(\lambda=275 \mathrm{~nm})$ intensity.

Interestingly, we observed a significant increase in the RI contrast of the waveguide channels following the polinginhibition step rather than a decrease as suggested in the literature. A comparison of the maximum RI contrasts for waveguides written with different UV laser intensities before and after the EFP step is shown in Fig 2. All of these waveguides were written using the same scanning speed $(0.1$ $\mathrm{mm} / \mathrm{s})$ and spot radius $(\sim 3 \mu \mathrm{m})$.

As seen in the plot of Fig. 2, a greater than three fold increase in the maximum RI contrast of the waveguide channels can be observed after the EFP step. The effect of RI contrast enhancement is additionally demonstrated by the observation of waveguide propagation after the EFP step in UV tracks written at intensities below $0.1 \mathrm{MW} / \mathrm{cm}^{2}$. The RI contrast provided by just UV irradiation (prior to the EFP step) was insufficient to support waveguide modes.

An explanation for the experimentally observed enhancement of the RI contrast can be provided by a RI discontinuity which occurs on the domain boundary as reported in Ref. 15 . According to this report the RI changes abruptly in the vicinity of the domain boundary. It decreases in the newly poled domain and increases suddenly in the unpoled part of the crystal on the opposite side of the domain boundary. This abrupt RI transition takes place within a width ranging from $2 \mu \mathrm{m}$ to $4 \mu \mathrm{m}$ and provides the appropriate RI profile to explain the observed enhancement of the RI contrast within the waveguide.

The ridge structures are finally produced in the last fabrication step which is wet etching in HF acid. The formation of the ridge structures results from the differential etching between the two opposite polar surfaces of LN in HF acid ${ }^{16}$. When the poled samples are etched in HF acid the sets of parallel domains with $+\mathrm{z}$ orientation act as etch-stop layers whereas the surrounding $-z$ face gradually etches down to reveal sets of parallel ridges.

Fig. 3 shows an optical microscope image of two sets of parallel ridges (each set consisting of four ridges corresponding to identical UV writing conditions) formed using this fabrication route. All of the ridges have been produced by scanning at a speed of $0.1 \mathrm{~mm} / \mathrm{s}$. However, each set of four ridges correspond to different UV laser intensity. The set on the left of the image has been written with an incident intensity of $\sim 0.17 \mathrm{MW} / \mathrm{cm}^{2}$ whereas, the set on the right has been written using a slightly lower intensity of $\sim 0.14 \mathrm{MW} / \mathrm{cm}^{2}$. A small difference in the writing intensity was observed to influence the width of the poling-inhibited region and hence the width of the corresponding ridges. The impact of the laser intensity on the width of the resulting 
ridges was studied systematically and the results are summarized in Fig.4. The figure shows a plot of the domain width, and hence that of the ridge structure as a function of the UV laser intensity which was used in the relevant fabrication step. As seen in Fig. 4 the widths of the poling inhibited domains are proportional to the UV laser intensity between $0.15 \mathrm{MW} / \mathrm{cm}^{2}$ to $0.2 \mathrm{MW} / \mathrm{cm}^{2}$ showing some evidence of saturation at higher intensities (above 0.2 $\left.\mathrm{MW} / \mathrm{cm}^{2}\right)$.

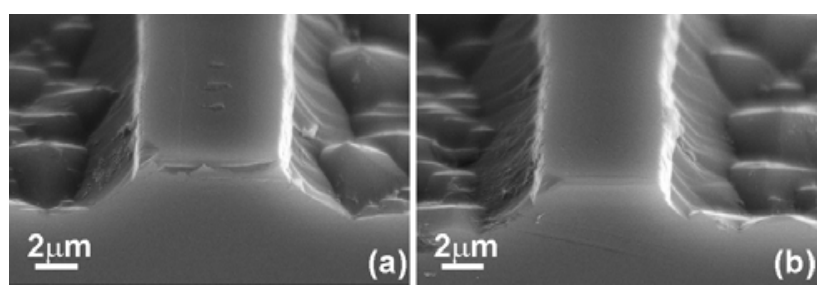

FIG. 5 SEM images of the polished end faces of two ridges fabricated with different UV laser intensities (SEM image acquired at $60^{\circ}$ tilt).

Scanning electron microscopy (SEM) images of the crosssections of ridges were obtained after mechanical polishing of the samples, and typical examples are presented in Fig. 5. The ridges in Fig 5(a) and 5(b) correspond to writing intensities of $0.17 \mathrm{MW} / \mathrm{cm}^{2}$ and $0.14 \mathrm{MW} / \mathrm{cm}^{2}$ respectively and the difference in their width resulting from this slight variation of the intensity is evident. The symmetric cross-sectional profile of the ridges is a manifestation of them being aligned to the crystallographic $y$ direction. The noticeable roughness of the planar surfaces (-z faces) on either sides of the ridge is typical of the etching of $-z$ faces in $\mathrm{HF}^{16}$. However, the sidewalls of the ridges are sufficiently free of this background surface roughness and hence are not expected to adversely affect the guided optical mode.

The UV laser intensity employed during the direct laser writing step also has a significant impact on the quality of the ridge structures. Specifically, UV intensities higher than $\sim 0.19 \mathrm{MW} / \mathrm{cm}^{2}$ lead to damage of the top-surface, whereas intensities lower than $\sim 0.13 \mathrm{MW} / \mathrm{cm}^{2}$ result in poorly defined ridge side-walls. However, there exists a definite range of intensity that corresponds to the optimum fabrication parameters. After mechanical polishing of the waveguide ends, laser light (633 nm HeNe laser) was end-fire coupled into the ridge waveguides using a microscope objective lens in order to investigate the optical characteristics of the structures. Fig. 6 shows the near-field intensity profile collected using a second objective lens that imaged the output face of the ridge on to a charge-coupled device (CCD) camera. This mode profile corresponds to a ridge structure formed using a UV laser intensity of $\sim 0.14 \mathrm{MW} / \mathrm{cm}^{2}$.

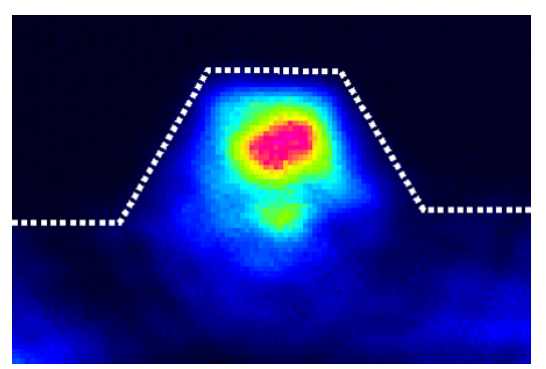

FIG. 6 Near-field intensity profile obtained at $633 \mathrm{~nm}$. The dashed line outlines the physical shape of the ridge structure.

In conclusion we have presented a method that utilizes a single UV-laser direct-writing step to both define the vertical confinement of the light in the waveguide and assist the domain engineering process (poling-inhibition) which, after chemical etching, produces the ridge super-structures. Moreover, EFP in conjunction with UV direct writing was observed to enhance the RI contrast which was initially inscribed by the UV laser irradiation. A much more detailed characterisation of this fabrication method would subsequently lead to further improvements and refinements. However, because of its ease of use and versatility the process has the potential to facilitate the fabrication of compact integrated optical devices in this very important optical ferroelectric material.

Financial support from the EU in the framework of the STREP033297 3D-DEMO is gratefully acknowledged.

${ }^{1}$ W. Sohler, H. Hu, R. Ricken, V. Quiring, C. Vannahme, H. Herrmann, D. Buchter, S. Reza, W. Grundkotter, S. Orlov, H. Suche, R. Nouroozi, and Y. Min, Optics \& Photonics News 19, 24, (2008).

${ }^{2}$ R. V. Schmidt and I. P. Kaminow, Appl. Phys. Lett. 25, 458, (1974). ${ }^{3}$ J. L. Jackel, C. E. Rice, and J. J. Veselka, Appl. Phys. Lett. 41, 607, (1982).

${ }^{4}$ I. P. Kaminow, Ramaswamy.V, R. V. Schmidt, and E. H. Turner, Appl. Phys. Lett. 24, 622, (1974).

${ }^{5}$ R. S. Cheng, W. L. Chen, and W. S. Wang, IEEE Photonics Technol. Lett. 7, 1282, (1995).

${ }^{6} \mathrm{H}$. Hu, R. Ricken, W. Sohler, and R. B. Wehrspohn, IEEE Photonics Technol. Lett. 19, 417, (2007).

${ }^{7}$ A. Guarino, G. Poberaj, D. Rezzonico, R. Degl'Innocenti, and P. Günter, Nat. Photonics 1, 407, (2007).

${ }^{8}$ K. Noguchi, O. Mitomi, H. Miyazawa, and S. Seki, Journal of Lightwave Technology 13, 1164, (1995).

${ }^{9}$ I. E. Barry, G. W. Ross, P. G. R. Smith, and R. W. Eason, Appl. Phys. Lett. 74, 1487, (1999).

${ }^{10}$ M. Iwai, T. Yoshino, S. Yamaguchi, M. Imaeda, N. Pavel, I. Shoji, and T. Taira, Appl. Phys. Lett. 83, 3659, (2003).

${ }^{11}$ S. Mailis, C. Riziotis, I. T. Wellington, P. G. R. Smith, C. B. E. Gawith, and R. W. Eason, Optics Letters 28, 1433, (2003).

${ }^{12}$ P. Ganguly, C. L. Sones, Y. J. Ying, H. Steigerwald, K. Buse, E. Soergel, R. W. Eason, and S. Mailis, Journal of Lightwave Technology 27, 3490, (2009).

${ }^{13}$ C. L. Sones, A. C. Muir, Y. J. Ying, S. Mailis, R. W. Eason, T. Jungk, A. Hoffmann, and E. Soergel, Appl. Phys. Lett. 92, 072905, (2008).

${ }^{14}$ M. de Angelis, S. De Nicola, A. Finizio, G. Pierattini, P. Ferraro, S. Grilli, and M. Paturzo, Appl. Phys. Lett. 85, 2785, (2004).

${ }^{15}$ V. Gopalan, V. Dierolf, and D. A. Scrymgeour, Ann. Rev. Mater. Res. 37, 449, (2007).

${ }^{16}$ C. L. Sones, S. Mailis, W. S. Brocklesby, R. W. Eason, and J. R. Owen, J. Mater. Chem. 12, 295, (2002). 